\title{
Two Algorithms of Power Loading for MCM System in Nakagami Fading Channel
}

\author{
Lev GOLDFELD, Vladimir LYANDRES, Lior TAKO \\ Department of Electrical and Computer Engineering, Communication Laboratory \\ Ben-Gurion University of the Negev, Beer-Sheva, Israel \\ Email: lyandres@ee.bgu.ac.il \\ Received November 19, 2009; revised December 22, 2009; accepted January 17, 2010
}

\begin{abstract}
We consider two non-iterative algorithms of adaptive power loading for multicarrier modulation (MCM) system, The first one minimizes the average power of the system transmitter and ensures the preset average bit-error rate, while the second reduces the average transmitting power subject to the given values of demanded bit-error rate and of the outage probability. The algorithms may be used for power-efficient management of the up-link in cellular communication, where mobile terminals use rechargeable batteries, or of the downlink in satellite communication with solar power source of a transponder. We present performance analysis of the adaptive MCM systems supported by computer simulation for the case of the $m$-Nakagami fading and additive white Gaussian noise in the forward and backward channels. Evaluation of the power gain of the proposed strategies and its comparison with uniform power loading shows that the gain depends on the fading depth and average signal to noise ratio in the system sub-channels.
\end{abstract}

Keywords: MCM, Fading Channel, Adaptive Power Loading

\section{Introduction}

Data transmission over bandwidth-limited channel using a multicarrier modulation (MCM) has attracted a lot of interest, due to its ability to achieve relatively good reception quality in spite of frequency selective fading. If the channel state information (CSI) is available at the transmitter side, the MCM system performance can be significantly improved by means of adaptive power loading (APL) [1-5].

In this paper, we propose two non-iterative APL strategies. The first strategy, relevant to the case of continuous data transmission, is to minimize the average transmitting power required for the given value the average bit-error rate (ABER). The purpose of the second strategy, more suitable for packet transmission, is to search for a value of the average transmitting power which is sufficient for given values of ABER and of the outage probability. The proposed strategies might be useful for power management of the uplink cellular communication channel, since mobile terminals use rechargeable batteries, or of the downlink from a satellite transponder with solar power sources.

At first, we assume that the communication channel is time-invariant, i.e. it has a "frozen" frequency-selective transfer function and suffers only from additive Gaussian noise. Under the assumption of the perfect channel state information we evaluate average power gain (APG) due to adaptation as a function of the Signal-to-Noise Ratio averaged over the system sub channels (ASNR). Although this premise is too optimistic, it is widely used in the literature while evaluating the upper bound for performance of the adaptive MCM system.

\section{Algorithms of Adaptive Power Loading}

We assume that the channel amplitude transfer function is well staircase approximated, i.e. it may be presented as a set of $N$ equal-bandwidth sub-channels, each with the same noise power $\sigma^{2}$ and with a constant random gain $K_{n}, n \in[1, N]$ known to the transmitter precisely. The ABER of the MCM system is defined as

$$
\bar{P}_{e r N}=\frac{1}{N} \sum_{n=1}^{N} p_{e r n}\left(\gamma_{n} P_{\operatorname{Tr} n}\right)
$$

where $p_{\text {er } n}$ is the bit-error rate in the $n$-th sub channel

$$
\gamma_{n}=K_{n}^{2} / \sigma^{2}
$$


is the normalized partial SNR and $P_{T r n}$ is the power loaded in the $n$th sub channel.

\subsection{The First Algorithm}

We define the normalized partial BER in the AMCM system as

$$
b_{n}=\frac{p_{e r n}}{N \cdot \bar{P}_{e r N}}
$$

It is evident that

$$
\sum_{n=1}^{N} b_{n}=1
$$

and the total transmitted power is written now as

$$
P_{\operatorname{TrN}}=\sum_{n=1}^{N} P_{\operatorname{Trn}}\left(b_{n}\right)
$$

The search of the optimal power partition vector

$$
\mathbf{P}_{\text {Tr N opt }}=\left\{P_{\text {Trn opt }}\right\}_{n=1}^{N}
$$

can be formulated as minimization of (5) subject to the required value of ABER $\bar{P}_{\text {er } N}=$ const and can be found by the LaGrange method, i.e. solving the system of equations:

$$
\frac{\partial\lfloor\Phi(\vec{b})]}{\partial\left(b_{n}\right)}=0, \quad n=1, \ldots, N,
$$

where

$$
\Phi(\vec{b})=P_{\operatorname{TrN}}(\vec{b})-\lambda\left(\sum_{n=1}^{N} b_{n}-1\right)
$$

and $\lambda$ is the LaGrange multiplier.

Let us consider, for example, the solution to this problem for the case of MPSK modulation in sub channels, for which BER in the $n$th sub channel is [7]

$$
p_{\text {er } n}=\frac{2 Q\left(\sqrt{2 \log _{2} M \cdot \gamma_{n} P_{\operatorname{Trn}}} \sin \pi / M\right)}{\log _{2} M}
$$

It is well known that $Q$-function may be approximated as

$$
Q(x)=a \exp \left(-x^{2} / a\right)
$$

where $a$ is the coefficient dependent on $x^{2}$. From (8) and (9), we obtain the following expression for the partial BER

$$
p_{\text {er } n} \approx \alpha_{1} \exp \left(-\alpha_{2} \gamma_{n} P_{T r n}\right)
$$

and using a simple transformation of (1) together with (3), the total transmitter power may be written as

$$
P_{T r N}=-\sum_{n=1}^{N} \frac{\ln \left(N \bar{P}_{e r N} b_{n} / \alpha_{1}\right)}{\alpha_{2} \gamma_{n}}
$$

where

$$
\alpha_{1}=2 a / \log _{2} M
$$

and

$$
\alpha_{2}=\frac{2 \log _{2} M \sin ^{2}(\pi / M)}{a}
$$

Substituting (11) into (6) and searching for $\min P_{\operatorname{Tr} N}$ subject to $\bar{P}_{e r N}=$ const, we obtain the optimal transmitter power partition

$$
P_{\text {Tr n opt }}=-\left(\alpha_{2} \gamma_{n}\right)^{-1} \ln \left[\frac{N \bar{P}_{e r N}}{\alpha_{1} \gamma_{n}}\left(\sum_{k=1}^{N} 1 / \gamma_{k}\right)^{-1}\right]
$$

The minimal total transmitting power $P_{\operatorname{Tr} N o p t}$ providing the given value of ABER of the MCM system is written as

$$
P_{T r N \text { opt }}=\sum_{n=1}^{N}-\frac{1}{\alpha_{2} \cdot \gamma_{n}} \ln \left(\frac{N \cdot \bar{P}_{\text {er } N}}{\alpha_{1}} \frac{1 / \gamma_{n}}{\sum_{k=1}^{N} 1 / \gamma_{k}}\right)
$$

\subsection{The Second Algorithm}

Let us assume that certain time invariant bounded value of the ABER is required

$$
P_{\text {erN }}=\frac{1}{N} \sum_{n=1}^{N} p_{\text {er } n}\left(\gamma_{n} P_{T r n}\right) \leq P_{\text {erbound }}
$$

and the average transmitting power is to be minimized. For the non adaptive MCM system the condition is reached for any value $K_{n} \geq K_{\min }$ if the equal power $P_{T r n}$ loaded into any sub-channels satisfies the following condition

$$
\gamma_{\text {min }}=P_{\text {erbound }}^{-1}(\gamma)=\frac{K_{\min }^{2} P_{T r n}}{\sigma_{n}^{2}}
$$

To achieve the requested value of BER in all sub channels of the AMCM system, the total power of the transmitter $P_{\operatorname{TrN}}\left(\bar{K}_{n}\right)$ should be distributed between sub channels in conformity with the following rule

$$
\begin{gathered}
P_{\operatorname{Tr} n}=P_{\operatorname{Tr} N}^{(\max )}=\frac{\left(K_{\min } / K_{n}\right)^{2}}{N} P_{T r N}^{(\max )} \text { for } K_{n} \geq K_{\min } \\
P_{\operatorname{Tr} n}=P_{\operatorname{Tr} n}^{(\text {test })}=\frac{P_{\operatorname{Tr} N}^{(\max )}}{N} \text { for } K_{n}<K_{\min }
\end{gathered}
$$


Subject to the equity of the total transmitting powers in the adaptive and in the system with uniform power loading of the sub-channels. If the condition $K_{n} \geq K_{\text {min }}$ is not satisfied for certain sub-channels, they are blocked, and instead a test signal for the sub-channels quality control is transferred ${ }^{1}$.

Communication reliability can be defined as the probability of this condition being satisfied in all sub-channels of the system

$$
R_{N}=\left(R_{n}\right)^{N}=\left(\int_{K_{\text {min }}}^{\infty} f_{K}(K) d K\right)^{N}=1-P_{\text {out }}
$$

where $K_{\text {min }}$ it the minimal value of the sub channel gain, that provides the required value of the BER for given $P_{\operatorname{TrN}}$ and $P_{\text {out }}$ is outage probability.

\section{Performance of the Adaptive MCM System in Fading Channel}

In this Section, we consider the performance of the proposed systems in a time-varying channel. We assume that fading processes in the sub channels are mutually independent and have the same $m$-Nakagami probability density function (PDF) [7] of the sub channels gain

$$
f_{K_{i}}\left(K_{i}\right)=\frac{2 m^{m}}{\Gamma(m) K_{0}^{2 m}} K_{i}^{2 m-1} \exp \left(-\frac{m K_{i}^{2}}{K_{0}^{2}}\right)
$$

where $m \geq 0.5$ and $K_{0}^{2}>0$ are parameters and $\Gamma($.$) is$ the Gamma function. The PDF (20) is able to span the entire range from the deepest one-sided Gaussian fading to no-fading conditions and it is widely used to fit experimental data obtained on a variety of propagation paths from HF sky wave transmission to UHF urban radio propagation and VHF satellite links [7].

Let us define the Average Power Gain (APG) of the AMCM system in fading channel as

$$
\eta_{N}(d B)=10 \lg \left(P_{\operatorname{TrN}}^{(U)} / \bar{P}_{\operatorname{Tr} N}\right)
$$

where $P_{\operatorname{Tr} N}^{(U)}$ is the total transmitted power of MCM-U system and $\bar{P}_{T r N}$ is the average transmitting power of the system with APL. Using Equations (15), (18), (19) and (20) we obtain, after certain transformations, the final expressions for APG. For the optimal APL

$$
\eta_{N}^{(1)}=\frac{2 \gamma_{0} \log _{2} M \cdot \sin ^{2}(\pi / M)}{a\left[\frac{2^{N-1} m^{0.5 m N+1}}{\Gamma^{N}(m)} A_{1}-\frac{m^{m}}{\Gamma(m)} \ln \frac{N \bar{P}_{e r N}}{2 a} A_{2}\right]}
$$

${ }^{1}$ This algorithm was mentioned under the name: Truncated Channel Inversion Power Loading Algorithm in [7]. In [5] the result of its use for minimization of error probability, and in [7] for maximization of the system capacity was investigated. where

$$
\begin{gathered}
A_{1}=\int_{0}^{\infty} y^{0.5 m-1} \ln (1+y)\left\{\int_{0}^{t} t^{0.5 m N-1} I_{m-2}(2 \sqrt{m t y}) K_{m}^{N-1}(2 \sqrt{m t}) d t\right\} d y \\
A_{2}=N T(m+1)(m)^{-(m+1)}
\end{gathered}
$$

$I_{k}($.$) is the modified Bessel function of the k$-th order and $K_{m}(\cdot)$ is the McDonald function of the $m$-th order.

For the second APL strategy APG is written as

$$
\eta_{N}^{(2)}=\frac{\Gamma(m)}{m \gamma_{0}^{2} \Gamma\left(m-1, m \gamma_{0}^{2}\right)+\gamma\left(m, m \gamma_{0}^{2}\right)}
$$

where $\gamma_{0}^{2}=\frac{K_{\min }^{2}}{K_{0}^{2}}, \Gamma(x, y)$ is incomplete gamma function and $\gamma(x, y)$ is alternative incomplete gamma function. For this case the equation for the outage probability $P_{\text {out }}$ may be obtained from (17) and (18):

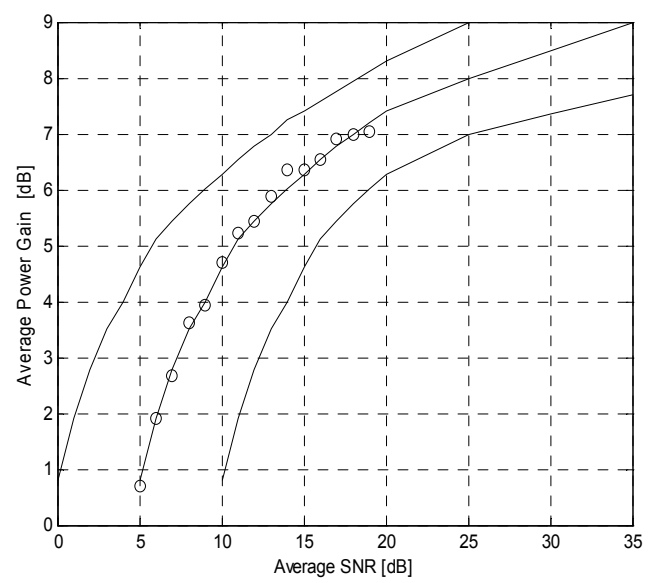

Figure 1. Average power gain of the first APL algorithm as a function of the average SNR.

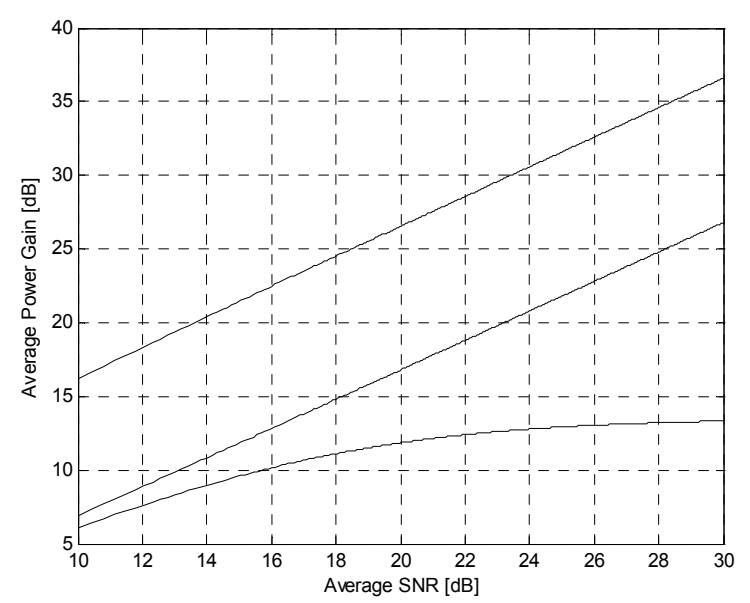

Figure 2. Average power gain of second APL algorithm as a function of the average SNR. 


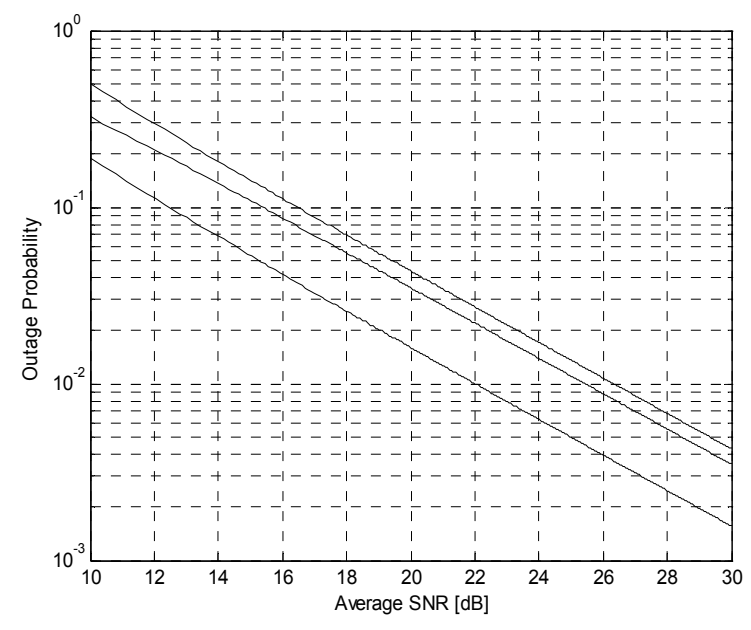

Figure 3. Outage probability of the second APL algorithm as a function of the average SNR.

$$
P_{\text {out }}=1-\left[\frac{\Gamma\left(m, m K_{\min }^{2} / K_{02}\right)}{\Gamma(m)}\right]^{N}
$$

The results of simulation and calculation of APG as a function of average normalized SNR is presented in Figure 1 for the first APL algorithm and in Figure 2 for the second one $(\mathrm{N}=16, \mathrm{~m}=0.75 ; 1 . ; 2)$. The results indicate that the gain increases, with increasing of average normalized SNR in sub channels (at the expense of increase $K_{0}^{2}=E\left\{K_{n}^{2}\right\}$ ). It is necessary to underline here, that as the depth of fading increases (lower $m$ ) the APG becomes more significant.

When comparing the gain values for the proposed strategies it is necessary to take into account that optimal APL algorithm (15)) provides minimum of average transmitting power at the same size of ABER that was obtained in MCM system with uniform power distribution (MCM-U). On the other hand the algorithm (18)) reduces the average power for a given values of BER and outage probability. Outage probability $P_{\text {out }}$ as a function of average normalized SNR is presented in Figure 3, from where it follows that $P_{\text {out }}$ decreases with increasing of average normalized SNR and increases for more deeply of fading (lower value of $m$ ).

\section{Conclusions}

In this letter, we investigate low-complexity (non-iterative) algorithms of power-adaptation of MCM system in slow $m$-Nakagami fading channel. The first of the algorithms is relevant to continuous data transmission and minimizes the average power of the system transmitter subject to the required value of the average BER. The second algorithm searches for such value of the transmitter power that is sufficient for the given values of the average BER and of the system outage probability. The gain in transmitting power increases with increasing of the fading depth, and of the average SNR. In subRayleigh and Rayleigh channels it can exceed $10 \mathrm{~dB}$.

\section{References}

[1] L. Goldfield, V. Lyandres and D. Wulich, "Minimum BER power loading for OFDM in fading channel," IEEE Transaction on communications, Vol. 49, pp. 14-18, January 2001.

[2] L. Goldfield, "Adaptive OFDM system in fading channel," European Transactions on Telecommunications, Vol. 14, pp. 293-300, February 2002.

[3] N.Y. Ermolova and B. Makarevich, "Power and subcarrier allocation algorithms for OFDM systems," in Proc. IEEE 16-th International Symposium on Personal, Indoor and Mobile Radio Communications, pp.352-356, 2005.

[4] L. Goldfeld and V. Lyandres "Minimum power loading strategy for multicarrier system in the capacity constrained frequency selective channel," Electronic Letters, Vol. 41, No. 25, pp. 1386-1388, December 2005.

[5] C. Mutti, D. Dahlhaus and T. Hunziker, "Optimal power loading for multiple-input single-output OFDM systems with bit level interleaving," IEEE Transaction on Wireless Communications, Vol. 5, pp. 1886-1895, May 2006.

[6] A. Goldsmith, "Wireless Communications", Cambridge University Press, 2005. 